\title{
UTILIZAÇÃO DE MEDICAMENTOS POR GESTANTES: UMA REVISÃO SISTEMÁTICA DA LITERATURA
}

USE OF MEDICINES BY PREGNANT: A SYSTEMATIC REVIEW OF THE LITERATURE

Lícia Kaira Pereira Silva ${ }^{a^{*}}$

Orcid: https://orcid.org/0000-0001-9361-6151

\author{
Ana Emilia Formiga Marques ${ }^{\mathbf{b}^{*}}$ \\ Orcid: https://orcid.org/0000-0003-1568-7231
}

licia-kaira@hotmail.com ${ }^{\mathrm{a}^{*}}$, anaemiliaformiga@hotmail.com ${ }^{\mathrm{b} *}$

Estácio FMJ - Juazeiro do Norte - CE*
Data de Submissão :22/05/2019

Data de Aceite: 21/10/2019

\section{RESUMO}

Introdução: A gravidez é período em que o organismo da mulher sofre grandes alterações, sendo a maioria dessas alterações não patológicas e em decorrência dessas alterações fisiológicas. Objetivo: Analisar a produção científica acerca do uso de medicamentos no período da gestação. Metodologia: Realizou-se uma revisão sistemática da literatura onde foram analisados artigos indexados nas bases de dados LILACS, SCIELO e MEDLINE com os descritores: Gestantes, Uso de medicamentos e Farmacoepidemiologia, no período entre 2013 e 2018. Resultados: A revisão sistemática foi estruturada por meio de 11 artigos e após a análise dos dados constatou-se que com exceção do ácido fólico, a maioria das gestantes relatadas nos estudos fez uso de pelo menos um medicamento, sendo os antibióticos os mais prescritos. A maior parte dos medicamentos utilizados são pertencentes a classes que o seu uso é considerado seguro durante a gravidez. Em relação à automedicação, observou-se que muitas mulheres gestantes fazem uso de medicamentos por contra própria para tratar sintomas comuns à gravidez, sendo o percentual mais alto de automedicação antes do início do pré-natal. Conclusão: Assim conclui-se que as gestantes apresentam desconhecimento dos riscos da automedicação e que o profissional farmacêutico é de fundamental importância no acompanhamento da gestante, visando prevenir possíveis complicações tanto na mãe quanto no feto.

Palavras-chave: Gestantes; uso de medicamentos; Farmacoepidemiologia.

\section{ABSTRACT}

Introduction: Pregnancy is a period in which the woman's body undergoes major changes, being most of these pathological changes and as a result of these physiological changes. Objective: Analyze the scientific literature about the use of medicines in the gestation period. Methods: we performed a systematic review of the literature where we analyzed articles indexed in the databases LILACS, SCIELO and MEDLINE with the keywords: pregnant women, Pharmacoepidemiology and Drug Use in the period between 2013 and 2018. Results: A systematic review was structured through 11 articles and after data analysis it was found that with the exception of folic acid, the majority of pregnant women reported in the studies made use of at least one medicine, being the the most prescribed antibiotics. Most of the medications used are from classes that your usage is considered safe during pregnancy. In relation to self-medication, it was observed that many pregnant women make use of medicines on your own to treat common symptoms to pregnancy, being the highest percentage of self-medication before the start of prenatal care. Conclusion: So it is concluded that pregnant women present ignorance of the risks of self-medication and that the professional pharmacist is of fundamental importance in the monitoring of pregnant women, in order to prevent possible complications in both the mother and the fetus.

Keywords: Pregnant women; use of medicines; Pharmacoepidemiology 


\section{Introdução}

A gravidez de uma mulher é período que seu organismo sofre grandes alterações, sendo que a maioria dessas alterações não são patológicas. Em decorrência das alterações fisiológicas que as mulheres sofrem durante a gestação, existe uma gama de medicamentos que são prescritos à mulher nessa fase. Sendo assim, o acompanhamento clínico de gestantes pelo farmacêutico torna-se indispensável devido ao elevado número de medicamentos utilizados ${ }^{1}$.

O que deve ser levado em conta é que a maioria dos medicamentos pode atravessar a barreira placentária, apresentando toxicidade ao bebê. Em relação aos medicamentos prescritos durante a gestação, os principais medicamentos prescritos durante a gravidez são: "sulfato ferroso $(53,2 \%)$, paracetamol $(9,7 \%)$, hioscina $(4,8 \%)$, metronidazol $(4,8 \%)$ e hidróxido de alumínio (3,6\%)."Nicaretta et al (2016) abordou que os AINES (anti-inflamatórios não esteroides) possuem uma gama de indicação clínica, tais como, "antipiréticos, analgésicos e anti-inflamatórios"2.

Esses fármacos só podem ser utilizados durante o período gestacional quando o risco benefício justificar o risco para o feto. Quanto ao uso de anti-inflamatórios corticosteroides, é necessário cautela em relação ao uso durante a gravidez, devido os glicocorticoides serem teratogênicos. "As tetraciclinas são antibióticos eficazes contra bactérias gram-positivas e gram-negativas, incluindo Rickettsias, Coxiella, Mycoplasma e Chamydia spp." Tais medicamentos, espalham-se por todo o tecido e também nos líquidos corporais, são lipossolúveis e atravessam a barreira placentária ${ }^{3}$.

Além da utilização dos fármacos com prescrição médica, é preocupante também o uso dos fármacos utilizados sem indicação médica, destacando-se o paracetamol, brometo de N-Butilescopolamina (Buscopan) e Dipirona. A classe terapêutica mais consumida sem prescrição médica foram os "analgésicos, antipiréticos e

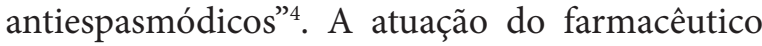
oferece uma oportunidade inovadora desempenhando um importante papel na equipe de profissionais de saúde. Assim, o médico e o farmacêutico podem trabalhar em parceria no aconselhamento e orientação da gestante em relação à medicação ${ }^{5}$.

Assim tem-se como objetivo geral analisar a produção científica acerca do uso de medicamentos no período da gestação através de uma revisão integrativa de literatura. O estudo tratou-se de uma revisão sistemática da literatura. A revisão da literatura contribui para as discussões sobre métodos e resultados de pesquisas, assim como reflexões sobre a realização de futuros estudos. A pesquisa foi realizada seguindo algumas etapas operacionais: identificação do tema e seleção da hipótese ou questão da pesquisa; estabelecimento de critérios para inclusão e exclusão de estudos/amostragem ou busca na literatura; definição das informações a serem extraídas dos estudos selecionados/ categorização dos estudos; interpretação dos resultados; e apresentação da revisão/síntese do conhecimento ${ }^{6}$.

A pesquisa foi direcionada pela seguinte questão norteadora: Quais as evidências científicas acerca da utilização de medicamentos por gestantes?

Para o levantamento dos dados, foram selecionados artigos das bases de dados: Literatura Latino-Americana e do Caribe em Ciências da Saúde (LILACS), Scientific Electronic Library Online (SCIELO) e Medical Literature Analysis and Retrieval System on-line (MEDLINE) com os descritores selecionados através do Decs: Gestantes, Uso de medicamentos e Farmacoepidemiologia. Para a seleção dos artigos, foram utilizados os seguintes critérios de inclusão: artigos completos e disponíveis em meio eletrônico; realizados entre 2013 e 2018; nos idiomas português, inglês ou espanhol; e que abordaram a temática. Foram excluídos artigos com publicações repetidas, revisões de literatura sem rigor metodológico, editoriais, manuais, dissertações e teses. O levantamento dos artigos foi realizado no período de agosto de 2018.

\section{Desenvolvimento}

A partir da combinação dos descritores foram obtidos 303 estudos. Numa avaliação inicial por meio dos resumos, verificou-se que 110 não abordavam o tema propriamente dito. 193 foram 
avaliados pelo resumo e pelo título sendo excluídos 82 por pesquisas serem revisão sistemática e em outras línguas. 111 foram selecionados para leitura completa sendo excluídos 100 pela não elegibilidade. Portanto, a revisão sistemática foi estruturada por meio de 11 artigos.
A análise dos dados foi realizada de forma descritiva. Os estudos foram reunidos em grupos, os quais permitiram avaliar as evidências, bem como identificar a necessidade de investigações futuras acerca da temática.

A Figura 1 abaixo sintetiza o processo de seleção dos artigos.

Figura 1 - Fluxograma do processo de seleção dos estudos

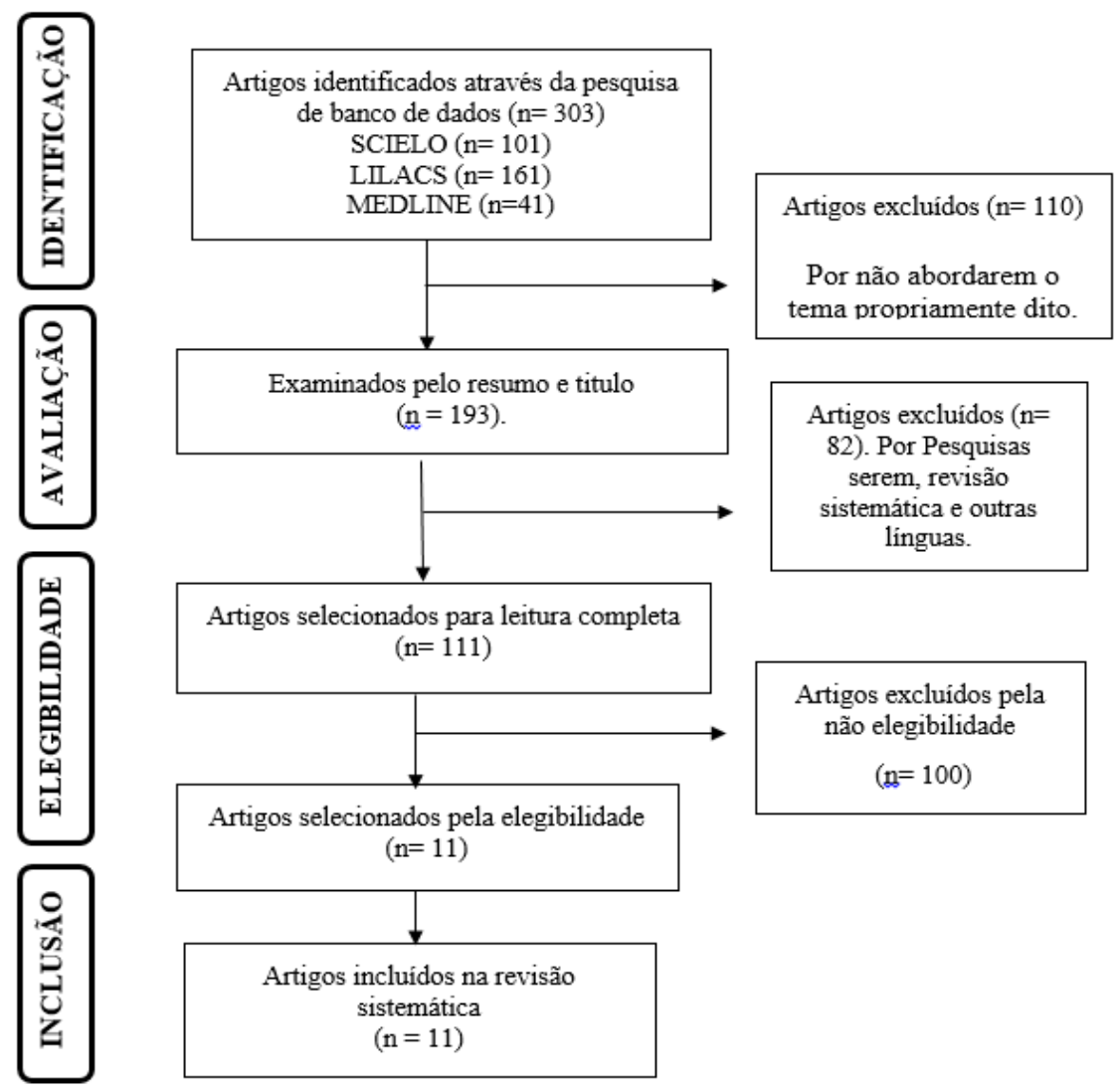

Conforme a busca eletrônica realizada, os artigos selecionados foram identificados por ordem cronológica. O Quadro 01 sintetiza os conteúdos das publicações selecionadas quanto ao autor e ano, título, revista e metodologia abordada. 
Quadro 01- Caracterização dos estudos científicos de acordo com o autor, ano, título, revista e metodologia.

\begin{tabular}{|c|c|c|c|}
\hline AUTOR/ANO & TITULO & REVISTA & METODOLOGIA \\
\hline $\begin{array}{l}\text { Lunardi, Schuelter, } \\
\text { Galato (2014) }\end{array}$ & $\begin{array}{c}\text { Uso de medicamentos no primeiro } \\
\text { trimestre de gravidez: avaliação da } \\
\text { segurança dos medicamentos e uso de } \\
\text { ácido fólico e sulfato ferroso }\end{array}$ & Rev Bras Ginecol Obstet. & Estudo transversal de coorte \\
\hline $\begin{array}{l}\text { Fritzen, Colet, } \\
\text { Oliveira (2014). }\end{array}$ & $\begin{array}{l}\text { Uso de antimicrobianos por gestantes no } \\
\text { serviço público de saúde. }\end{array}$ & Rev Bras Promoç Saúde. & $\begin{array}{l}\text { Estudo transversal analítico e } \\
\text { retrospectivo. }\end{array}$ \\
\hline Barral et al (2014) & $\begin{array}{l}\text { Risk factors of hiv-1 vertical } \\
\text { transmission (vt) and the influence of } \\
\text { antiretroviral therapy (art) in pregnancy } \\
\text { outcome. }\end{array}$ & Rev. Inst. Med. trop. S. Paulo & Estudo transversal de coorte \\
\hline Mothupi (2014) & $\begin{array}{l}\text { Use of herbal medicine during } \\
\text { pregnancy among women with access } \\
\text { to public healthcare in Nairobi, Kenya: a } \\
\text { cross-sectional survey. }\end{array}$ & $\begin{array}{l}\text { BMC Complementary and } \\
\text { Alternative Medicine }\end{array}$ & Estudo transversal de coorte \\
\hline Dillon et al (2015) & $\begin{array}{l}\text { Prevalence of prescribing in pregnancy } \\
\text { using the Irish primary care research } \\
\text { network: a pilot study. }\end{array}$ & $\begin{array}{l}\text { BMC Pregnancy and } \\
\text { Childbirth. }\end{array}$ & $\begin{array}{l}\text { Estudo transversal analítico e } \\
\text { retrospectivo. }\end{array}$ \\
\hline Tinker et al (2015) & $\begin{array}{l}\text { Prevalence of Prescription Medication } \\
\text { Use among Non-Pregnant Women of } \\
\text { Childbearing Age and Pregnant Women } \\
\text { in the United States - NHANES, } 1999 \\
-2006\end{array}$ & $\begin{array}{l}\text { Maternal and child health } \\
\text { jornal }\end{array}$ & $\begin{array}{l}\text { Estudo transversal analítico e } \\
\text { retrospectivo. }\end{array}$ \\
\hline $\begin{array}{l}\text { Yamamoto et al } \\
\text { (2015). }\end{array}$ & $\begin{array}{l}\text { Disparities in antidepressant use in } \\
\text { pregnancy. }\end{array}$ & $\begin{array}{l}\text { Journal of Perinatology: } \\
\text { Official Journal of the } \\
\text { California Perinatal } \\
\text { Association }\end{array}$ & Estudo transversal de coorte \\
\hline $\begin{array}{l}\text { Kassada et al } \\
\qquad(2015)\end{array}$ & $\begin{array}{c}\text { Prevalência e fatores associados ao uso } \\
\text { de drogas em gestantes atendidas em } \\
\text { atenção primária. }\end{array}$ & Texto contexto - enferm. & Estudo analítico transversal \\
\hline $\begin{array}{l}\text { Hernández et al } \\
\text { (2017) }\end{array}$ & $\begin{array}{c}\text { Prescripción de metildopa en } \\
\text { el tratamiento de pacientes con } \\
\text { enfermedad hipertensiva en el embarazo. }\end{array}$ & MEDISAN & Estudo descritivo transversal \\
\hline $\begin{array}{l}\text { Campoverde et al } \\
\qquad(2017)\end{array}$ & $\begin{array}{c}\text { PTI y embarazo: Una experiencia con } \\
\text { Rituximab. }\end{array}$ & Rev Chil Obstet Ginecol & Relato de experiência \\
\hline $\begin{array}{l}\text { Costa, Coelho, } \\
\text { Santos (2017) }\end{array}$ & $\begin{array}{c}\text { Utilização de medicamentos antes e } \\
\text { durante a gestação: prevalência e fatores } \\
\text { associados. }\end{array}$ & Cad. Saúde Pública & Estudo transversal de coorte. \\
\hline
\end{tabular}

Fonte: Dados da pesquisa, 2018

O Quadro 02 sintetiza os conteúdos das

e principais conclusões.

publicações selecionadas quanto ao título, objetivo 
Quadro 02- Caracterização dos estudos científicos de acordo com o título, objetivo e principais conclusões.

\begin{tabular}{|c|c|c|}
\hline TÍTULO & OBJETIVO & PRINCIPAIS CONCLUSÕES \\
\hline $\begin{array}{l}\text { Uso de medicamentos no primeiro } \\
\text { trimestre de gravidez: avaliação da } \\
\text { segurança dos medicamentos e uso } \\
\text { de ácido fólico e sulfato ferroso. }\end{array}$ & $\begin{array}{c}\text { Identificar o perfil de uso de medicamentos } \\
\text { no primeiro trimestre de gravidez }\end{array}$ & $\begin{array}{l}\text { No momento do diagnostico da gravidez há } \\
\text { um reduzido número de uso de medicamentos, } \\
\text { observa-se maior consumo no desenvolver da } \\
\text { gestação. }\end{array}$ \\
\hline $\begin{array}{l}\text { Uso de antimicrobianos por } \\
\text { gestantes no serviço público de } \\
\text { saúde. }\end{array}$ & $\begin{array}{l}\text { Identificar e classificar quanto ao risco os } \\
\text { antimicrobianos prescritos para gestantes }\end{array}$ & $\begin{array}{l}\text { A maioria dos antimicrobianos prescritos na } \\
\text { gestação apresenta risco B o que significa que } \\
\text { não há evidência direta de risco ao concepto. }\end{array}$ \\
\hline $\begin{array}{l}\text { Risk factors of hiv-1 vertical } \\
\text { transmission (vt) and the influence } \\
\text { of antiretroviral therapy (art) in } \\
\text { pregnancy outcome. }\end{array}$ & $\begin{array}{l}\text { Analisar os fatores de risco da transmissão } \\
\text { vertical do HIV-1 em gestantes soropositivas }\end{array}$ & $\begin{array}{l}\text { Apesar dos possíveis riscos de uso de ARV } \\
\text { pela mãe durante a gravidez, os benefícios } \\
\text { para o recém-nascido são compensatórios } \\
\text { e seu uso não influenciou o resultado da } \\
\text { gravidez. }\end{array}$ \\
\hline $\begin{array}{l}\text { Use of herbal medicine during } \\
\text { pregnancy among women with } \\
\text { access to public healthcare in } \\
\text { Nairobi, Kenya: a cross-sectional } \\
\text { survey. }\end{array}$ & $\begin{array}{l}\text { Determinar o padrão de uso de fitoterapia } \\
\text { em um ambiente urbano, onde as mulheres } \\
\text { têm relativamente alto acesso à saúde } \\
\text { pública. }\end{array}$ & $\begin{array}{l}\text { A medicina herbal é usada por } 12 \% \text { das } \\
\text { mulheres grávidas com acesso a cuidados } \\
\text { de saúde e muitas vezes ocorre sem o } \\
\text { conhecimento dos profissionais de saúde. }\end{array}$ \\
\hline $\begin{array}{l}\text { Prevalence of prescribing in } \\
\text { pregnancy using the Irish primary } \\
\text { care research network: a pilot study. }\end{array}$ & $\begin{array}{l}\text { Estabelecer a prevalência e os padrões de } \\
\text { prescrição para mulheres grávidas em um } \\
\text { ambiente de atenção primária irlandesa. }\end{array}$ & $\begin{array}{l}\text { A prevalência global de prescrição para } \\
\text { mulheres grávidas nesta pesquisa é baixa em } \\
\text { comparação com estudos internacionais. }\end{array}$ \\
\hline $\begin{array}{l}\text { Prevalence of Prescription } \\
\text { Medication Use among Non- } \\
\text { Pregnant Women of Childbearing } \\
\text { Age and Pregnant Women in the } \\
\text { United States - NHANES, } 1999 \text { - } \\
2006 .\end{array}$ & $\begin{array}{c}\text { Examinar o uso de medicamentos prescritos } \\
\text { entre mulheres grávidas e não grávidas nos } \\
\text { Estados Unidos. }\end{array}$ & $\begin{array}{l}\text { Medicamentos para alergia e doenças } \\
\text { infecciosas foram mais comuns entre as } \\
\text { gestantes. Uso de medicação por prescrição } \\
\text { para asma e distúrbios da tireoide foi relatado } \\
\text { por ambos os grupos. }\end{array}$ \\
\hline $\begin{array}{c}\text { Disparities in antidepressant use in } \\
\text { pregnancy }\end{array}$ & $\begin{array}{l}\text { Determinar a frequência de uso de } \\
\text { medicação antidepressiva relatada durante } \\
\text { as consultas de ambulatório para mulheres } \\
\text { grávidas. }\end{array}$ & $\begin{array}{l}\text { Na maioria dos casos as mulheres gestantes } \\
\text { relatavam episódios de depressão, tornando } \\
\text { os medicamentos antidepressivos os mais } \\
\text { prescritos. }\end{array}$ \\
\hline $\begin{array}{l}\text { Prevalência e fatores associados } \\
\text { ao uso de drogas em gestantes na } \\
\text { atenção primária. }\end{array}$ & $\begin{array}{l}\text { Identificar a prevalência do uso de drogas } \\
\text { por gestantes atendidas na atenção básica. }\end{array}$ & $\begin{array}{l}\text { Um grande número de gestantes faz uso de } \\
\text { pelo menos um medicamento sem prescrição } \\
\text { médica e os medicamentos mais utilizados são } \\
\text { os antianêmicos, seguidos pelos antieméticos } \\
\text { e analgésicos. }\end{array}$ \\
\hline $\begin{array}{l}\text { Prescripción de metildopa en } \\
\text { el tratamiento de pacientes con } \\
\text { enfermedad hipertensiva en el } \\
\text { embarazo. }\end{array}$ & $\begin{array}{c}\text { Caracterizar a prescrição de metildopa em } \\
\text { gestantes. }\end{array}$ & $\begin{array}{l}\text { O uso da metildopa prevaleceu em pacientes } \\
\text { que apresentavam situações associadas } \\
\text { às formas mais graves da doença, como } \\
\text { idade avançada, nuliparidade e história de } \\
\text { hipertensão arterial. }\end{array}$ \\
\hline
\end{tabular}




\begin{tabular}{|c|c|l|}
\hline TÍTULO & OBJETIVO & PRINCIPAIS CONCLUSÕES \\
\hline $\begin{array}{c}\text { PTI y embarazo: Una experiencia } \\
\text { con Rituximab. }\end{array}$ & $\begin{array}{c}\text { Descrever a experiência no tratamento de } \\
\text { uma grávida no segundo trimestre com } \\
\text { tratamento para Púrpura trombocitopenia } \\
\text { idiopática com corticosteroides. }\end{array}$ & $\begin{array}{l}\text { O uso de Rituximab melhorou } \\
\text { substancialmente a contagem de plaquetas, } \\
\text { reduziu o risco de hemorragias graves na mãe. }\end{array}$ \\
\hline $\begin{array}{c}\text { Utilização de medicamentos antes } \\
\text { e durante a gestação: prevalência e } \\
\text { fatores associados. }\end{array}$ & $\begin{array}{c}\text { Avaliar a prevalência e os fatores associados } \\
\text { àtilização de medicamentos em gestantes } \\
\text { antes e durante a gravidez }\end{array}$ & $\begin{array}{l}\text { Em sua maior parte, os medicamentos } \\
\text { utilizadosantes do diagnóstico dagravidez,por } \\
\text { automedicação, são os relaxantes musculares, } \\
\text { analgésicos como dipirona e paracetamol e } \\
\text { contraceptivos orais combinados. }\end{array}$ \\
\hline
\end{tabular}

Fonte: Dados da pesquisa, 2018

Após a leitura e interpretação dos 11 artigos selecionados para a Revisão sistemática da literatura, os resultados foram dispostos de acordo com a classificação de risco, utilizando-se os cinco grupos de risco definidos pelo Food and Drug Administration (FDA).

Excluindo o ácido fólico, a maioria das gestantes relatadas nos estudos fez uso de pelo menos um medicamento ${ }^{7,8}$. Os antibióticos e o salbutamol foramosmedicamentosmais prescritos ${ }^{9,10}$, seguidos do paracetamol e antidepressivos ${ }^{11}$, fitoterápicos ${ }^{12}$, antimicrobianos $^{8,13}$ e contraceptivos ${ }^{5,7,9}$. Em relação à classificação de risco, a maior parte dos medicamentos utilizados pelas gestantes pertence às classes $\mathrm{A}$ e $\mathrm{B}$, categorias sem risco para o feto, seguidos da classe C e X. Não foi encontrado nenhum relato do uso de medicamentos da classe D.

Quadro 03 - Classificação de risco definidos pelo FDA.

\begin{tabular}{|c|c|}
\hline Categoria & Medicamentos \\
\hline A & Ácido fólico, Vitamina A, Sulfato ferroso \\
\hline B & $\begin{array}{r}\text { Paracetamol, Amoxilina, Metronidazol, } \\
\text { Salbutamol, Insulina. }\end{array}$ \\
\hline C & Metildopa, Rituxmab. \\
\hline D & - \\
\hline X & Contraceptivos \\
\hline
\end{tabular}

Fonte: Dados da pesquisa, 2018

Entre as mulheres grávidas, a medicação mais comumente relatada foi a levotiroxina, medicamento que é usado para tratar distúrbios da tireoide. Além da amoxicilina em consonância com os autores supracitados. Também constatouse o uso de nitrofurantoína, antibiótico utilizado para o tratamento de cistite e uretrite bacteriana e a combinação de sulfametoxazol e trimetoprima ${ }^{10}$.

Certos medicamentos para asma e alergia também estavam entre os medicamentos de prescrição mais comumente relatados, especificamente albuterol (salbutamol), montelucaste, cetirizina, budenoside e prometazina, o último dos quais é mais comumente prescrito para náuseas e vômitos. A insulina também esteve entre os 10 medicamentos mais frequentemente relatados nos últimos 30 dias entre gestantes ${ }^{9,10}$.

A prevalência do uso de medicamentos para tratar condições como a asma não tratada e o hipotireoidismo revela a importância de se estudar o perfil de segurança desses medicamentos para mulheres grávidas e como consequência um melhor manejo dessas situações na gestação ${ }^{11}$.

Outra classe de medicamentos utilizada durante a gestação são os antidepressivos. Em outro estudo o uso de antidepressivos foi relatado durante 2,2\% de todas as consultas ambulatoriais para mulheres grávidas. Os profissionais constataram um diagnóstico de depressão em $4,5 \%$ das visitas. Entre as consultas voltadas para gestantes com depressão, os profissionais relataram uso de antidepressivos em $25,4 \%$ das vezes em todas as visitas ${ }^{12}$.

Os antimicrobianos estão também entre os medicamentos utilizados durante a gestação. Em estudo realizado para avaliar o uso de antimicrobianos por gestantes verificou- 
se a prescrição destes medicamentos em acompanhamento pré- natal. Miconazol e metronidazol creme vaginal, cefalexina $500 \mathrm{mg}$, azitromicina $500 \mathrm{mg}$ e amoxicilina $500 \mathrm{mg}$ foram os mais prescritos ${ }^{8,13}$.

O uso da metildopa também foi constatado na literatura. Um estudo foi realizado em Cuba em relação ao uso deste medicamento em pacientes com doença hipertensiva durante a gravidez. O que foi constatado além da alta prevalência, foi a polifarmácia que deveu-se principalmente à utilização de suplementos pré-natais, ou seja, a combinação de dose fixa de dois comprimidos prescritos como suplemento para a prevenção de deficiências nutricionais específicos durante a gestação, contendo quatro drogas: fumarato ferroso (100 mg), ácido fólico $(0,25 \mathrm{mg})$, ácido ascórbico (150 mg) e vitamina A (20 000 $\mathrm{U})$; suplemento que foi prescrito em todos os membros do estudo ${ }^{8,13}$.

Diferente dos outros estudos já relatados, um estudo transversal incluiu 333 mulheres que frequentavam uma clínica de cuidados infantis em um hospital distrital de saúde pública em Nairóbi, no Quênia, avaliou o uso da medicina herbal como automedicação, evidenciando elevada prevalência. As gestantes usavam fitoterapia para dor nas costas, dor de dente, indigestão e doenças infecciosas, como infecções do trato respiratório e malária. O uso dos fitoterápicos ainda gera muitas dúvidas e discute-se que os profissionais de saúde devem desempenhar um papel no uso racional da medicina herbal e ocidental, orientando sobre as contraindicações e os riscos de interações medicamentosas com os usuários ${ }^{12}$.

Em relação à automedicação, observou-se que muitas mulheres gestantes fazem uso de medicamentos por contra própria para tratar sintomas comuns a gravidez. Em estudo realizado com 212 gestantes encontrou-se um percentual de $46,7 \%$ estavam em uso de medicamentos no momento do diagnóstico dagravidez. O percentual mais alto de automedicação ocorreu antes do início do pré-natal. Na época do diagnóstico da gravidez, observou-se expressivo uso de medicamentos que atuam no sistema geniturinário e hormônios sexuais, como anticoncepcionais orais, o que provavelmente está relacionado ao percentual de gestações não planejadas ${ }^{7}$.

Em se tratando de efeitos adversos, apenas dois estudos relataram preocupação em relação ao tratamento durante a gravidez. Em estudo realizado com gestantes em uso de Terapia antirretroviral (TAR), a realização desse tratamento durante a gravidez não apresentou associação com baixo escore no quinto minuto de Apgar. Apesar dos possíveis riscos de uso da TAR pela mãe durante a gravidez, os benefícios para o recém-nascido são compensatórios e seu uso não influenciou o resultado da gravidez ${ }^{15,16}$. Desfechos indesejáveis como baixo peso ao nascer, escore de apgar abaixo de sete no quinto minuto e parto prematuro parecem estar relacionados à realização de prénatal inadequado e ao comportamento materno ${ }^{15}$.

Outro estudo realizado com gestantes portadoras de Púrpura Trombocitopênica Idiopática(PTI) evidenciou que ouso de Rituximab, melhorou substancialmente a contagem de plaquetas e reduziu o risco de hemorragias graves na mãe. Não observou-se grandes complicações maternas e o resultado neonatal aos 4 meses de nascimento foi satisfatório, sem detectar leuco ou trombocitopenia durante sua evolução ${ }^{16}$.

$\mathrm{O}$ autor supracitado ainda complementa que embora haja pouca experiência em pacientes grávidas, o Rituximab é uma droga que deve ser considerada como uma alternativa com segurança e eficácia aceitáveis no manejo da segunda linha em gestantes com $\mathrm{PTI}^{16}$.

\section{Conclusão}

Observou-se que durante a gestação, excluindo o ácido fólico, a maioria das gestantes fez uso de pelo menos um medicamento, sendo os antibióticos os mais prescritos. A maior parte dos medicamentos utilizados pertence às classes A e B, ou seja, o seu uso é considerado seguro durante a gravidez. Em relação à automedicação, observou-se que muitas mulheres gestantes fazem uso de medicamentos por contra própria para tratar sintomas comuns a gravidez, sendo o percentual mais alto de automedicação ocorreu antes do início do pré-natal, concluindo- se que as gestantes apresentam desconhecimento dos riscos da automedicação.

Analisando os dados encontrados acerca do uso de medicamentos durante a gravidez, podese inferir que o profissional farmacêutico é de fundamental importância no acompanhamento da 
gestante, visando prevenir possíveis complicações tanto na mãe quanto no feto. Assim, o farmacêutico enquanto profissional promovedor da saúde tem a responsabilidade de acompanhar a gestante evitando o uso irracional de medicamentos.

\section{Referências}

1. Roque, ATF; Carraro TE. Narrativas sobre a experiência de ser puérpera de alto risco. Revista Escola Anna Nery. 2015;19(2):272-278.

2. Brum Lucimar Filot da Silva, Pereira Patricia, Felicetti Lilian Leticia, Silveira Renata Dischke da. Utilização de medicamentos por gestantes usuárias do Sistema Único de Saúde no município de Santa Rosa (RS, Brasil). Ciênc. saúde coletiva [Internet]. $2011 ; 16(5$ ): 2435-2442.

3. Richter, F., Rigo, M., Castro, L., Kauffmann, C. and Ely, L. (2020). Utilização de medicamentos por gestantes usuárias do sistema único de saúde em um município do vale do Taquari - RS.

4. Nascimento Áurea M do, Gonçalves RELM, Medeiros RMK, Lisboa HCF. Avaliação do uso de medicamentos por gestantes em unidades básicas de saúde de Rondonópolis, Mato Grosso. Rev. G\&S [Internet]. 17(1):Pág. 96-112.

5. Costa Débora Bomfim, Coelho Helena Lutescia Luna, SantosDjanilson Barbosa dos. Utilização demedicamentos antes e durante a gestação: prevalência e fatores associados. Cad. Saúde Pública [Internet]. 2017 [cited 2020 Jan 13] ; 33( 2 ): e00126215. Available from: http:// www.scielo.br/scielo.php?script $=$ sci_arttexd $=\mathrm{S} 0102$ 311X2017000205005\&lng=en. Epub Mar 09, 2017. http://dx.doi.org/10.1590/0102-311x00126215.

6. Soares Cassia Baldini, Hoga Luiza Akiko Komura, Peduzzi Marina, Sangaleti Carine, Yonekura Tatiana, Silva Deborah Rachel Audebert Delage. Revisão integrativa: conceitos e métodos utilizados na enfermagem. Rev. esc. enferm. USP [Internet]. 2014 Apr [cited 2020 Jan 13] ; 48( 2 ): 335-345. Available from: http:// www.scielo.br/scielo.php?script $=$ sci_arttexd $=\mathrm{S} 0080$ 62342014000200335\&lng=en. http://dx.doi.org/10.1590/ S0080-6234201400002000020.

7. Lunardi-Maia Tânia, Schuelter-Trevisol Fabiana, Galato Dayani. Uso de medicamentos no primeiro trimestre de gravidez: avaliação da segurança dos medicamentos e uso de ácido fólico e sulfato ferroso. Rev. Bras. Ginecol. Obstet. [Internet]. 2014 Dec [cited 2020 Jan 13] ; 36( 12 ): 541-547. Available from: http:// www.scielo.br/scielo.php?script $=$ sci_arttexd $=\mathrm{S} 0100$ 72032014001200541\&lng=en. http://dx.doi.org/10.1590/ So100-720320140005051.
8. Kassada Danielle Satie, Miasso Adriana Inocenti, Waidman Maria Angélica Pagliarini, Marcon Sonia Silva. Prevalência e fatores associados ao uso de medicamentos por gestantes atendidas na atenção primária. Textocontexto - enferm.[Internet].2015 Sep ; 24( 3 ): 713-721.

9. Dillon P, O’Brien K, McDonnell R, Donnelly-Swift E, Galvin R, Roche A et al. Prevalence of prescribing in pregnancy using the Irish primary care research network: a pilot study. 2020 .

10. Tinker, Sarah C., et al. "Prevalence of prescription medication use among non-pregnant women of childbearing age and pregnant women in the United States: NHANES, 1999-2006." Maternal and child health journal 19.5 (2015): 1097-1106.

11. Yamamoto A, McCormick MC, Burris HH. Disparidades no uso de antidepressivos na gravidez. Journal of Perinatology: Jornal Oficial da Associação Perinatal da Califórnia. Abr 2015; 35 (4): 246-251. DOI: 10.1038 / jp.2014.197

12. Mothupi, Mamothena Carol. Use of herbal medicine during pregnancy among women with access to public healthcare in Nairobi, Kenya: A cross-sectional survey. BMC complementary and alternative medicine. 2014. $14: 432$.

13. Fritzen, J. S., Colet, C. F., \& de Oliveira, K. R. . Uso de antimicrobianos por gestantes no serviço público de saúde. Revista Brasileira em Promoção da Saúde, 2014: 27(2), 198-206.

14. Ramos Hernández Leonardo, Soyet Medina Kenia, Rams Aguilera Ronaldo, Ramos Medina Javier L, Galves Martí Lilian Luisa. Prescripción de metildopa en el tratamiento de pacientes con enfermedad hipertensiva en el embarazo. MEDISAN ; 2017 Ago ; 21( 8 ): 960-968

15. Barral M, Oliveira G, Lobato R, Mendoza-Sassi R, Martínez A, Gonçalves C. Risk factors of hiv-1 vertical transmission (vt) and the influence of antiretroviral therapy (art) in pregnancy outcome. Revista do Instituto de Medicina Tropical de São Paulo. 2014;56(2):133-138.

16. Campoverde, P., Cuéllar, E., Miranda, J. P., Farías, M. L., \& Rojas, A.. PTI y embarazo: Una experiencia con Rituximab. Revista chilena de obstetricia y ginecología, 2017: 82(1), 80-83.

\section{Como citar este artigo:}

Silva LKP, Marques AEF. Utilização de medicamentos por gestantes: uma revisão sistemática da literatura. Rev. Aten. Saúde. 2019; 17(62): 90-97. 